\title{
Computational study of the interaction between natural rubber $a-$ terminal groups and L-quebrachitol, one of the major components of natural rubber
}

\author{
Joan Gimenez-Dejoz ${ }^{1} \cdot$ Katsuhiko Tsunoda ${ }^{2} \cdot$ Yasuo Fukushima $^{2} \cdot$ Keiji Numata $\rrbracket^{1,3}$
}

Received: 10 August 2021 / Revised: 24 September 2021 / Accepted: 26 September 2021 / Published online: 29 October 2021

(c) The Author(s) 2021. This article is published with open access

\begin{abstract}
Natural rubber is a biomaterial with unique physical and chemical features that are indispensable for many industrial applications. It is widely accepted that the $\alpha$-terminal groups of its biopolymer molecules play a critical role in its exceptional characteristics. Herein, we used molecular dynamics to model recently structurally defined $\alpha$-terminal groups and their interaction with L-quebrachitol, which is the second most common compound found in natural rubber particles.
\end{abstract}

\section{Introduction}

Natural rubber is a biopolymer of industrial importance because of its unique chemical and physical properties [1, 2]. It has high elasticity, resilience, and resistance to abrasion and impact, among other advantages. Therefore, it finds indispensable use in many fields and products, such as the production of tires for vehicles and planes and medical devices. Natural rubber is a secondary metabolite produced by different plant species, but most plants offer very low yields of rubber or low-molecular-weight polymers, affecting its properties [2]. In that context, the almost exclusive source of commercially viable raw biomaterial is extracted from the rubber tree Hevea brasilensis. Moreover, the commercialized synthetic rubber materials available through petrochemical industry derivatives do not possess the unique features, especially strain-induced crystallization behaviors, of natural rubber [3], making

Supplementary information The online version contains supplementary material available at https://doi.org/10.1038/s41428021-00569-w.

Keiji Numata

numata.keiji.3n@kyoto-u.ac.jp

1 Biomacromolecules Research Team, RIKEN Center for Sustainable Resource Science, Saitama, Japan

2 Advanced Materials Division, Bridgestone Corporation, Tokyo, Japan

3 Department of Material Chemistry, Graduate School of Engineering, Kyoto University, Kyoto, Japan substitution challenging in many applications. The poor properties of existing synthetic rubber can ultimately be attributed to a lack of understanding of the molecular mechanism of natural rubber biosynthesis, its structural and molecular characteristics and the composition of its alternative secondary constituents.

Natural rubber is composed mainly of high-molecularweight polymeric chains of cis-1,4-polyisoprene. This isoprene is created by a membrane-bound enzyme, cys-prenyl transferase (EC 2.5.1.20), located at the surface of cytoplasmic rubber particles and microscopic particles in the cytosol of $\mathrm{H}$. brasilensis, where rubber is compartmentalized [3]. The quality of natural rubber is dependent on several factors, with the high molecular weight of the polyisoprene chains and its stereoregularity regarded as the most important ones. In addition, other factors also include the composition of other elements present in the rubber $[4,5]$ and the structures of its terminal groups in the rubber chains [6]. Recently, Oouchi et al. [7] studied the terminal groups of natural rubber, suggested to be of paramount importance to the mechanical properties of rubber, and determined its structure.

The most abundant component after cis-1,4-isoprene rubber particles is the cyclic polyol L-quebrachitol [8], with an estimated abundance of $\sim 1.2 \%(\mathrm{w} / \mathrm{v})$ in $\mathrm{H}$. brasiliensis latex compared to sucrose, which has been estimated to be $\sim 0.4 \%(\mathrm{w} / \mathrm{v})$ [9]. The role of L-quebrachitol in rubber or in its production is still not clear, but several sugar transporters have been identified in specialized cells for rubber production (laticifiers) in $H$. brasiliensis [10], and there is evidence that high amounts of sugars are imported into laticifer cells [11]. Furthermore, the expression levels of this transporter are upregulated after treatment with ethylene 


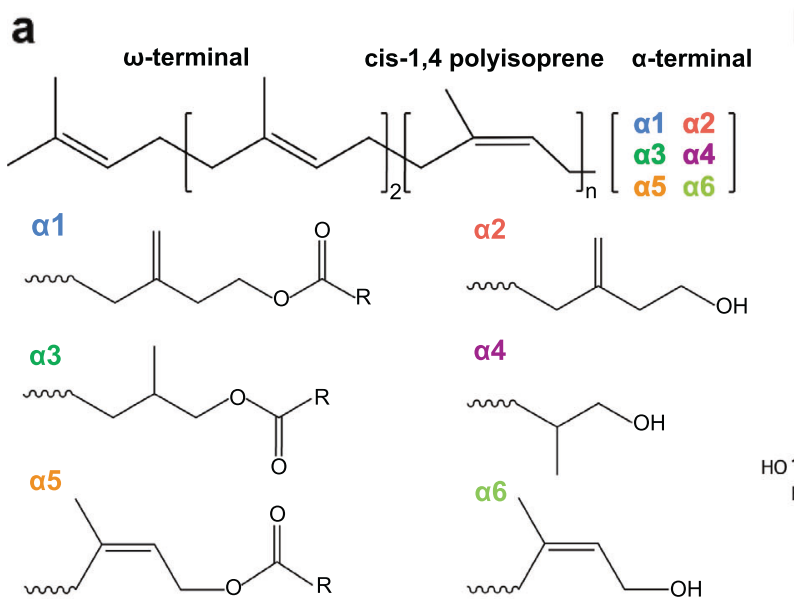

b
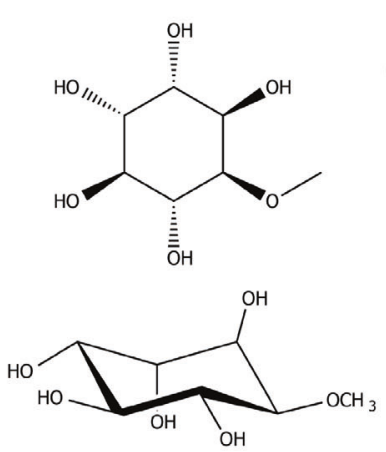

C

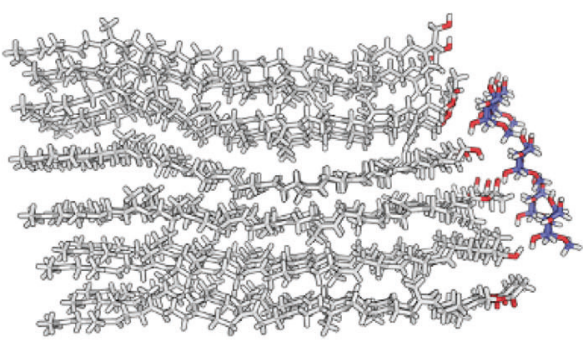

Fig. 1 Schematic representation of the systems modeled. a Schematic representation of the chemical structure of natural rubber showing the omega-terminal, the polyisoprene repeated unit ( $n=6$ in this study) and the distinct $\alpha$-terminal structures suggested by Oouchi et al. b Chemical structure of L-quebrachitol in top and chair representation. $\mathbf{c}$
Representation of the initial conformation of the simulated systems, with 18 rubber molecules (carbon atoms in gray sticks) located close together. Five L-quebrachitol molecules (carbon atoms in blue sticks) are positioned close to the $\alpha$-terminal groups. Oxygen and hydrogen atoms are displayed in red and white, respectively
[10]. Treatment with ethylene is a common practice used to increase the production of latex [12]. Although there is still no direct evidence of the involvement of L-quebrachitol in rubber synthesis, it is known that it has an important role in maintaining an increase in osmotic pressure in the cells and maintaining and increasing turgor pressure, which is essential for latex outflow [13]. However, the molecular mechanisms of L-quebrachitol or its interaction with rubber are still unknown; furthermore, due to its abundance in rubber particles and probable interplay with the isoprene molecules, it is important to understand how these components interact with one another, increasing the knowledge of the molecular rubber structure. Moreover, the role of the $\alpha$-terminal groups of rubber is poorly understood, and its interactions with other components in rubber particles are even less well understood. Therefore, we modeled the interaction of L-quebrachitol with rubber molecules with specific $\alpha$-terminal groups using atomistic molecular dynamics simulations. Our objective was to study the possible interaction of quebrachitol with rubber and whether this interaction can differ between different $\alpha$-terminal groups. Thus, this study could be useful for future rubber applications or improvement of synthetic rubber.

\section{Materials and methods}

\section{System construction}

We manually constructed rubber monomer molecules based on the study of Oouchi et al. using GaussView16. Six different monomers were constructed with the omegaterminal group, in addition to six repeats of the cis-1,4isoprene group and one of the six different $\alpha$-terminal groups suggested in the study of Oouchi et al. [7] (Fig. 1a). The molecules were parametrized using an antechamber [14] of AmberTools21 [15] at the AM1 semiempirical level and assigned AMBER GAFF atom types.

The L-quebrachitol molecule was constructed using GaussianView (Fig. 1b), energy minimization and geometry optimization were performed with quantum mechanics at Hartree-Fock/6-31G, and the restraint electrostatic potential (RESP) charges were calculated on the Hartree-Fock/6-31 $\mathrm{G}^{*}$ basis set with Gaussian16 [16]. The Antechamber module in AMBER20 [15] was used to RESP fit the calculated potentials to generate amber files.

Systems construction included one system with 18 monomer molecules, with 3 molecules of each $\alpha$-terminal type (Fig. 1c). In addition, 6 systems contained 12 molecules of only one of each $\alpha$-terminus. All rubber molecules were oriented with their $\alpha$-termini oriented at the same side, stacked closed together. The systems were constructed using the tleap program including Amber Tools20 [15] using the GAFF2 force field [17]. In all systems, 5 L-quebrachitol molecules were added near the $\alpha$-terminal groups of the molecules. All systems were created to run under vacuum conditions, and additional systems, created exactly from the same initial structures and force field as the vacuum systems, were solvated using TIP3P water molecules with a $10 \AA$ minimum distance between any solute atom and the edge of the periodic box. 
Fig. 2 Image of representative snapshots of the simulation trajectory of rubber with 1-quebrachitol. Rubber molecules are displayed in a gray surface and stick representation with oxygen atoms in red, and L-quebrachitol molecules are represented with blue spheres
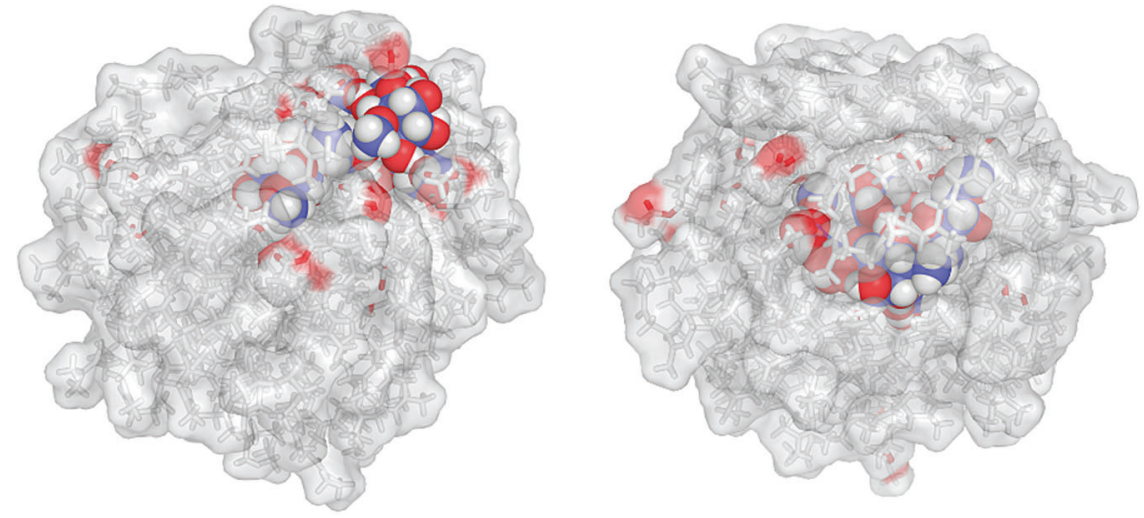

The systems were run using AMBER18 in vacuum or in a TIP3P water box. A time step of $2 \mathrm{fs}$ was used along with the SHAKE algorithm [18], while the particle-mesh Ewald (PME) method was used to calculate long-range interactions, with a cutoff of $12 \AA$. The systems were energy-minimized for 20,000 steps using the conjugate gradient minimization algorithm; then, they were slowly heated using the Langevin dynamic algorithm with a collision frequency gamma $=0.001$ $\mathrm{ps}^{-1}$ to $300 \mathrm{~K}$ over $600 \mathrm{ps}$ with the atoms fixed with a $10-\mathrm{kcal}$ $\mathrm{mol}^{-1}$ constraint. The solvated systems were equilibrated for $300 \mathrm{ps}$ in the NPT ensemble to equilibrate the pressure with the Berendsen barostat with all solute atoms restrained with $2 \mathrm{kcal}$ $\mathrm{mol}^{-1}$. Productive simulations were conducted for 260-300 ns for both explicit simulations and systems in vacuum. Analysis of the simulations was carried out with CPPTRAJ [19] analysis simulation software including amber tools.

\section{Results}

To analyze the possible role of L-quebrachitol in rubber, we constructed a small rubber system and analyzed the interactions of the molecules by molecular dynamics. Based on the recent work of Oouchi et al. [7], the individual rubber molecules consisted of three main parts: one side of the rubber molecule containing an omega-terminal rubber, a middle internal part consisting of six isoprene unit repeats, and the other end with the six $\alpha$-terminal groups (Fig. 1c shows the rubber scheme with L-quebrachitol). According to a previous study [7], six $\alpha$-terminal groups can be found in natural rubber, and we constructed and parametrized rubber molecules with each of the different $\alpha$-terminal groups (Fig. 1a). To model the highly hydrophobic environment expected to be found in natural rubber particles without water molecules, we constructed our systems using a small group of short rubber molecules with fewer isoprene units than in natural rubber and simulated them under vacuum conditions. We selected this methodology with short rubber molecules because simulating high-molecular- weight rubber molecules with a molecular weight of hundreds of thousands of $\mathrm{Da}$ at a high concentration of rubber molecules would be extremely computationally demanding, and vacuum conditions could provide an appropriate model for the hydrophobic heart of a rubber molecule. In total, 7 systems were constructed: one with 3 rubber molecules of each $\alpha$-terminus for a total of 18 rubber molecules and then systems containing 18 rubber molecules with one specific $\alpha$-terminal group (named a1 to a6) stacked closely together with the $\alpha$-terminal groups oriented in the same direction as a simplified model of the natural rubber structure. In addition, all systems contained 5 molecules of L-quebrachitol located on the surface of the rubber near its $\alpha$-terminal groups (Fig. 1c). After equilibration, each system was equilibrated and simulated for more than $250 \mathrm{~ns}$, allowing time to differ from the original structures and explore the energy landscape. We decided to include the $\alpha-6$ terminal group in the study, although its presence was not clearly determined in a previous study [7].

Using atomistic molecular dynamics, we explored how Lquebrachitol interacts with rubber molecules. In all systems, L-quebrachitol rapidly made contact with rubber and bound to its surface. After a few nanoseconds, the L-quebrachitol molecules were surrounded by rubber molecules and were internalized completely inside a rubber particle, where they remained for the rest of the simulation time (Fig. 2). To quantify the degree of internalization of L-quebrachitol within the rubber molecules, we calculated the exposed surface area (the surface area of a biomolecule that is accessible to a solvent) of all L-quebrachitol molecules for the simulation time (Table 1). This quantification of the exposed surface area confirmed that L-quebrachitol was almost completely surrounded by rubber molecules, as the exposed surface area of L-quebrachitol was below $5 \AA^{2}$ in all systems. For comparison, the calculated exposed surface area of L-quebrachitol when simulated in pure water was $330.8 \pm 2.1 \AA^{2}$ (Table 1). This suggests that L-quebrachitol could be present in hydrophobic rubber particles. We performed additional simulations of the same systems using 
explicit solvents, including water molecules (Supplementary Fig. S1). Due to the hydrophobic nature of the rubber, the rubber molecules rapidly compacted in the simulations, excluding the water molecules outside. In addition, the calculated exposed surface area (Table 2) indicated that due to its hydrophilic nature, L-quebrachitol did not interact with the rubber molecules. This was an expected result due to the hydrophobic and hydrophilic natures of rubber and Lquebrachitol, respectively. Rubber particles are isolated from the cytosol by a lipid-protein membrane, with the polyisoprene chain enclosed inside, which makes the particle core very hydrophobic [6]. In addition, we did not observe any difference in the interaction of L-quebrachitol and rubber with different $\alpha$-terminal groups (Table 2).

We investigated how the L-quebrachitol molecules interacted with the rubber molecules. Visual inspection of the simulation trajectories revealed that L-quebrachitol was surrounded by rubber particles in all vacuum systems and that $\mathrm{L}-$ quebrachitol clustered together. The molecules are found to

Table 1 Mean exposed surface area of all L-quebrachitol molecules and standard deviation in simulations in vacuum for the systems containing only one $\alpha$-terminal rubber $(\alpha 1-\alpha 6)$, the system containing 3 molecules of each $\alpha$-terminal rubber $(\alpha 1-\alpha 6)$ and for a molecule of L-quebrachitol (as a reference for all exposed surface area)

\begin{tabular}{lc}
\hline System & Surface area $\left(\AA^{2}\right)$ \\
\hline$\alpha 1$ & $3.5 \pm 1.4$ \\
$\alpha 2$ & $3.7 \pm 2.1$ \\
$\alpha 3$ & $4.6 \pm 2.7$ \\
$\alpha 4$ & $1.4 \pm 0.5$ \\
$\alpha 5$ & $2.5 \pm 1.1$ \\
$\alpha 6$ & $1.8 \pm 0.9$ \\
$\alpha 1-\alpha 6$ & $2.0 \pm 1.8$ \\
L-quebrachitol & $330.8 \pm 2.1$ \\
\hline
\end{tabular}

make H-bond interactions with other L-quebrachitol molecules and occasionally with $\alpha$-terminal groups of rubber, with their methyl groups oriented preferentially toward the rubber isoprene groups. Thus, we observed that they interacted preferentially with the $\alpha$-terminal groups of water molecules to avoid the hydrophobic isoprene groups of rubber. We further hypothesized that the molecules could interact differently with different $\alpha$-terminal groups, even if the interaction they displayed with the rubber molecules was similar (Table 1). Thus, we quantified the number of contacts L-quebrachitol molecules have during the simulation time, in which a contact is defined as a L-quebrachitol molecule located at less than $3 \AA$ of an $\alpha$ terminal group of a rubber molecule. Figure 3 clearly reveals a pattern in the number of contacts for the distinct rubber with different $\alpha$-termini. L-quebrachitol makes between 1 and 2 contacts (molecules are located in close proximity, less than 3 $\AA$ ) from an $\alpha$-terminal group of a rubber molecule in systems with $\alpha 1-, \alpha 3$ - and $\alpha 5$-termini, which have an ester group in their $\alpha$-terminus. However, in systems $\alpha 2, \alpha 4$ and $\alpha 6$, which have an alcohol group in their $\alpha$-terminus, the number of

Table 2 Mean exposed surface area of all L-quebrachitol molecules and standard deviation in simulations performed in water for the systems containing only one $\alpha$-terminal rubber $(\alpha 1-\alpha 6)$ and the system containing 3 molecules of each $\alpha$-terminal rubber $(\alpha 1-\alpha 6)$

\begin{tabular}{ll}
\hline System & Surface area $\left(\AA^{2}\right)$ \\
\hline$\alpha 1$ & $329.9 \pm 1.0$ \\
$\alpha 2$ & $322.7 \pm 0.5$ \\
$\alpha 3$ & $322.9 \pm 1.9$ \\
$\alpha 4$ & $322.7 \pm 1.2$ \\
$\alpha 5$ & $331.8 \pm 1.0$ \\
$\alpha 6$ & $321.7 \pm 2.0$ \\
$\alpha 1-\alpha 6$ & $325.9 \pm 1.6$ \\
\hline
\end{tabular}
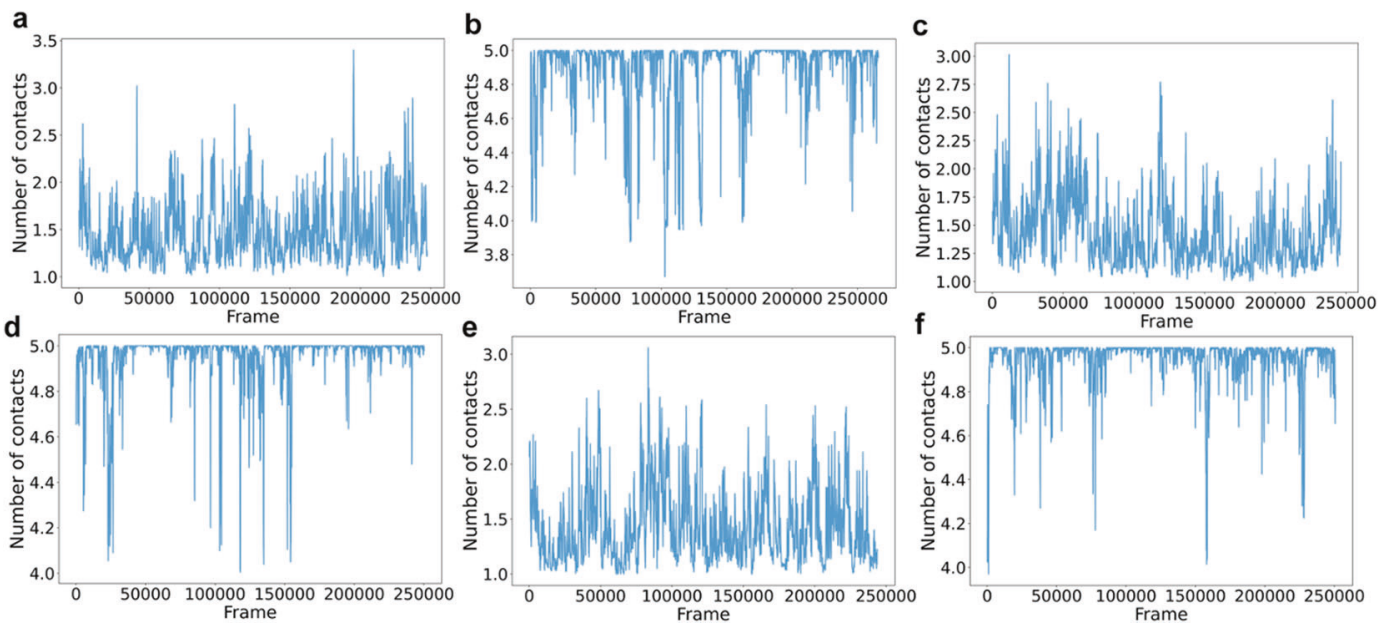

Fig. 3 Contacts between L-quebrachitol and rubber molecules with different $\alpha$-terminal groups, from $\alpha 1$ (a) to $\alpha 6$ (f) in order, over the trajectory frames (1000 frames represent one ns) 
contacts was higher during all simulation times, showing a number of contacts higher than 4 . This means that more Lquebrachitol molecules are located in closer proximity to the rubber $\alpha$-terminal molecules that have an alcohol group $(\alpha 2, \alpha 4$ and $\alpha 6$ ) compared with rubber molecules with an $\alpha$-terminus having an ester group ( $\alpha 1, \alpha 3$ and $\alpha 5)$. Therefore, we conclude that rubber molecules with $\alpha$-termini having an alcohol group can interact more easily with L-quebrachitol molecules, possibly impacting the physical properties of rubber particles, although the $\alpha$-terminal groups could also interact with nonrubber constituents by H-bonding or ionic interactions [20].

In summary, the analysis of the simulations shows that rubber and L-quebrachitol can interact in the highly hydrophobic environment of rubber particles. Moreover, although the L-quebrachitol molecules display a similarly low level of surface exposed area with all rubbers independent of their $\alpha$-terminal groups, from the individual analysis of the contacts, we can infer distinct interactions. Lquebrachitol clearly shows a higher number of contacts with rubber molecules with $\alpha$-termini having an alcohol group in their structure. This reinforces the idea of the importance of the specific $\alpha$-terminal groups on rubber and how subtle differences in its $\alpha$-terminal groups could impact its unique physical and chemical properties [7]. A better understanding of the molecular interactions of the rubber components will allow the synthesis of synthetic rubbers with better properties resembling those of natural rubber.

Acknowledgements We acknowledge the RIKEN Advanced Center for Computing and Communication (ACCC) for providing access to HOKUSAI BigWaterfall Supercomputer resources. This work was financially supported by Japan Technology Agency (JST) CREST (Grant no., JPMJCR2091), Grant-in-Aid for Transformative Research Areas (B), and JST Exploratory Research for Advanced Technology (JST-ERATO; Grant no., JPMJER1602).

\section{Compliance with ethical standards}

Conflict of interest The authors declare no competing interests.

Publisher's note Springer Nature remains neutral with regard to jurisdictional claims in published maps and institutional affiliations.

Open Access This article is licensed under a Creative Commons Attribution 4.0 International License, which permits use, sharing, adaptation, distribution and reproduction in any medium or format, as long as you give appropriate credit to the original author(s) and the source, provide a link to the Creative Commons license, and indicate if changes were made. The images or other third party material in this article are included in the article's Creative Commons license, unless indicated otherwise in a credit line to the material. If material is not included in the article's Creative Commons license and your intended use is not permitted by statutory regulation or exceeds the permitted use, you will need to obtain permission directly from the copyright holder. To view a copy of this license, visit http://creativecommons. org/licenses/by/4.0/.

\section{References}

1. Cornish K, Xie W. Natural rubber biosynthesis in plants: rubber transferase. Methods Enzymol. 2012;515:63-82.

2. Yamashita S, Takahashi S. Molecular mechanisms of natural rubber biosynthesis. Annu Rev Biochem. 2020;89:821-51.

3. Men X, Wang F, Chen GQ, Zhang HB, Xian M. Biosynthesis of natural rubber: current state and perspectives. Int $\mathrm{J}$ Mol Sci. 2019:20:50.

4. Monadjemi S, McMahan C, Cornish K. Effect of non-rubber constituents on Guayule and Hevea rubber intrinsic properties. J Res Updat Polym Sci. 2016;5:87-96.

5. Junkong P, Cornish K, Ikeda Y. Characteristics of mechanical properties of sulphur cross-linked guayule and dandelion natural rubbers. RSC Adv. 2017;7:50739-52.

6. Cherian S, Ryu SB, Cornish K. Natural rubber biosynthesis in plants, the rubber transferase complex, and metabolic engineering progress and prospects. Plant Biotechnol J. 2019;17:2041-61.

7. Oouchi M, Ukawa J, Ishii Y, Maeda H. Structural analysis of the terminal groups in commercial Hevea natural rubber by 2D-NMR with DOSY filters and multiple-WET methods using ultrahighfield NMR. Biomacromolecules. 2019;20:1394-1400.

8. Bealing FJ. Quebrachitol synthesis in Hevea-Brasiliensis. J Rubber Res Instit Malays. 1981;29:111-22.

9. Bealing FJ. Carbohydrate metabolism in Hevea latex-availability and utilization of substrates. J Rubber Res Inst Malays. 1969;21:445-55.

10. Dusotoit-Coucaud A, Porcheron B, Brunel N, Kongsawadworakul $\mathrm{P}$, Franchel J, Viboonjun U, et al. Cloning and characterization of a new polyol transporter (HbPLT2) in Hevea brasiliensis. Plant Cell Physiol. 2010;51:1878-88.

11. Bouteau F. Evidence of multiple sugar uptake across the plasma membrane of laticifer protoplasts from Hevea. Bioelectrochem Bioenerg. 1999;48:135-9.

12. D'auzac J. Ethylene, a new agent stimulating latex production in Hevea Brasiliensis. C R Hebd Seances Acad Sci Ser D. 1969;268:3046-9.

13. Sheldrake AR. Effects of osmotic stress on polar auxin transport in Avena mesocotyl sections. Planta. 1978;145:113-7.

14. Wang J, Wang W, Kollman PA, Case DA. Automatic atom type and bond type perception in molecular mechanical calculations. $\mathrm{J}$ Mol Graph Model. 2006;25:247-60.

15. Case DA, Aktulga HM, Belfon K, Ben-Shalom IY, Brozell SR, Cerutti DS, et al. Amber 2021. University of California, San Francisco (2021).

16. Frisch, MJ, Trucks, GW, Schlegel, HB, Scuseria, GE, Robb, MA, Cheeseman, JR, et al. Gaussian 16, Revision C.01. Gaussian, Inc., Wallingford CT (2016).

17. Wang J, Wolf RM, Caldwell JW, Kollman PA, Case DA. Development and testing of a general amber force field. J Comput Chem. 2004;25:1157-74.

18. Ryckaert JP, Ciccotti G, Berendsen HJC. Numerical integration of the cartesian equations of motion of a system with constraints: molecular dynamics of n-alkanes. J Comput Phys. 1977;23:327-41.

19. Roe DR, Cheatham TE. PTRAJ and CPPTRAJ: software for processing and analysis of molecular dynamics trajectory data. $\mathbf{J}$ Chem Theory Comput. 2013;9:3084-95.

20. Kitaura T, Kobayashi M, Tarachiwin L, Kum-ourm H, Matsuura A, Fushihara K, et al. Characterization of natural rubber end groups using high-sensitivity NMR. Macromol Chem Phys. 2018;219:1-11. 